\title{
Organisation and Reorganisation of Blood Vessels in Embryonic Development
}

\author{
V. NAVARATNAM \\ Cambridge
}

\begin{abstract}
Summary
The initial establishment of blood vessels is genetically predetermined but it is subsequently supplemented by local and haemodynamic factors. The earliest embryonic vessels arise independently in the mesenchyme of the yolk sac wall, chorion and embryonic disc. The angioblasts aggregate as cords which canalise by a process which includes intracellular vacuolation. Subsequent extension of the network occurs by a combination of (1) in situ formation of new vessels, (2) sprouting and fusion of established neighbouring vessels and (3) invading angioblasts from other regions. As capillaries mature, a basement membrane is established containing collagen IV, laminin, nidogen/entactin, heparan sulphate proteoglycan and a variable amount of fibronectin. Early capillary basement membrane contains more fibronectin which promotes proliferative and migratory activity of endothelium. As vessels mature, the endothelium switches to laminin secretion which causes differentiation including cell attachment, junction formation and establishment of tubes while cell proliferation is suppressed.
\end{abstract}

It is instructive to compare the factors affecting the differentiation of early embryonic vessels, which develop de novo from mesenchyme, with those influencing neovascularisation in the adult. Most of my comments will concern vasculature in general but I shall conclude this short communication by referring to certain vascular features of the retina where the influence of developmental factors is manifest.

In human development, the earliest vessels appear during the third week of gestation and, by the onset of the fourth week, the heart has started pulsating and an effective circulation has been established. Early endotheliallylined vessels appear in the mesoderm of the yolk sac, particularly in the plane immediately adjacent to the endodermal lining of the sac, and also at various sites within the embryonic disc and in the chorion. Yolk sac vessels were regarded as the most significant of these early sites (for instance, they are the most likely to contain differentiating haemoblasts in the lumen) and the view prevailed well into the twentieth century that intraembryonic vessels were formed as an extension of previously established yolk sac vessels. However, that view was challenged when Reagan ${ }^{1}$ demonstrated intraembryonic capillary formation even after connection between extraembryonic and intraembryonic tissues had been eliminated in prevascular chick embryos.

The initial establishment of blood vessels seems to be genetically predetermined and there is no doubt that they arise independently as angioblasts at several sites within the 
embryo and on extraembryonic membranes. The angioblastic cells contact each other forming cords along the course of future blood vessels. The cords progressively canalise (see below) and subsequent extension of the network occurs by a combination of in situ formation of endothelially lined vesicles, sprouting from established vessels and fusion of the various elements; sprouting involves the proliferation and migration of endothelial cells. Although Reagan's experiments and other evidence showed that intraembryonic vessels can arise in situ independent of extraembryonic angiogenesis, they did not exclude the possibility that invading angioblasts could supplement the local elements. Recent experiments by Noden, ${ }^{2}$ using transplants of prevascular mesoderm between different species, showed by appropriate antibody labelling that invading angioblasts did contribute to local vessel formation; however, when donor and recipient sites were mismatched, the developing vessels were appropriate to the host region thus indicating that control over blood vessel assembly resides in the local mesoderm and not in the invading cells.

Hereditary factors, supplemented by local influences such as hypoxia and mechanical features, remain significant in vessel formation for a surprisingly long period. For instance it has been demonstrated that establishment of vascular patterns, even of principal arteries and veins, continues for substantial periods even after the heart has been excised ${ }^{3,4}$ or stilled by the addition of chemicals such as potassium chloride or alcohol. ${ }^{5,6}$ Eventually, however, vessels are predominantly influenced by mechanical or haemodynamic influences in the circulation. In older embryos and adults, new vessel growth generally occurs only by endothelial sprouting from existing vessels but exceptions have been described of new vessels appearing in situ and then connecting to the circulation e.g. in bone marrow ${ }^{7}$ and retina. ${ }^{8}$

\section{Differentiation of endothelial cells, basement membrane formation and establishment of lumen}

Developing angioblasts are distinctly separated at first but there is progressive apposition, including formation of membrane junctions, to form cords. Three possible processes have been suggested for the canalisation of these cords:

(1) the lumen is formed (intercellularly) by separation of adjacent cells

(2) death of central cells in a cord

(3) formation of intracellular vacuoles which coalesce with each other and with the plasma membrane at opposite ends of the cell. ${ }^{9}$

There is evidence to suggest that intracellular vacuolation is at least a contributory mechanism; for instance ultrastructural studies indicate that the tips of capillary sprouts are 'seamless' (i.e. without cell junctions in cross section) and intracellular vacuoles have been demonstrated at the tips of sprouts in tissue culture experiments. ${ }^{10}$ It is interesting to note that the initial capillary endothelium does not exhibit a basement membrane. The cells start to secrete sulphated glycoproteins pari passu with the decline of non-sulphated protein synthesis which is proportional to cell mass. Initially the glycoproteins compose a glycocalyx on the luminal and abluminal aspects of the cell. As the cells flatten, however, a discontinuous basement membrane can be visualised on the abluminal surface. Already this is fairly late; for instance, in chick embryos it can be discerned no earlier than 48 hours after the heartbeat starts. A continuous basement membrane, which seems to signal stability of vessels, is not seen till even later; some authorities claim that it is established only after hatching.

\section{Role of basement membrane}

The view that stability of blood vessels is principally attributable to the presence of a basement membrane is supported by the fact that one of the earliest steps in neovascularisation is dissolution of this membrane. ${ }^{11,12}$ Endothelial cells maintain a highly polarised cytoplasm and the basolateral surface membrane contains distinctive proteins. The main structural constituent of endothelial basement membrane is collagen IV which binds the other components; the latter include the glycoproteins laminin and nidogen/entactin, heparan sulphate proteoglycan and a variable amount of fibronectin depending on the stage of development (see Table I). 
Table I Basement membrane components

\begin{tabular}{lll}
\hline Component & $M W$ & Possible roles \\
\hline Collagen IV & $540 \mathrm{~K}$ & attachment, differentiation \\
Laminin & $800 \mathrm{~K}$ & attachment, differentiation \\
Nidogen/Entactin & $150 \mathrm{~K}$ & adhesion \\
Heparan sulphate proteoglycan & $750 \mathrm{~K}$ & filtration, attachment \\
Fibronectin & $440 \mathrm{~K}$ & migration, proliferation \\
\hline
\end{tabular}

When labelled with appropriate antibodies, intraembryonic capillaries in six-day chick embryos were found to be associated with fibronectin, which seems to promote proliferative and migratory activity. Laminin secretion is not observed until days 8 to 10 when the vessels have a more stable appearance ${ }^{13}$ this switch to laminin secretion causes differentiation, including attachment and formation of junctions and establishment of endothelial tubes, while cell proliferation is suppressed. These views on the roles of basement membrane components are supported by observations of endothelial cell culture which attach well to laminin, fibronectin and various collagens: When rat microvascular cells are cultured on interstitial collagens I and III or on fibronectin, then proliferation is greatly enhanced over differentiation. By contrast, cells on laminin or on collagen IV exhibit attachment but proliferation is suppressed. Grant et al. ${ }^{13}$ have used a model system of human umbilical vein endothelial cells, which rapidly form capillary-like tubes when plated on reconstituted basement membrane gel, and they applied antibodies to inhibit various basement membrane components. They thus identified two separate components on laminin side chains, which have been sequenced and cloned, with the abilities to mediate cellto-matrix adhesion (RGD sequence) and tube formation (YIGSR sequence).

\section{Origin of periendothelial elements}

Perivascular mesenchymal cells express smooth muscle-specific $\alpha$-actin, and development of larger vessels from the capillary network proceeds by the apposition of such cells. Information on their further differentiation is perfunctory but it appears that the outer coats of blood vessels comprising pericytes, fibrocytes and smooth muscle develop from these elements.

\section{Vascular development in the retina}

Detailed study of development of retinal vessels may throw some light on capillary growth under pathological conditions. The mammalian retina differentiates from an invaginated neuroepithelium which becomes stratified to form the familiar cellular and plexiform layers. The layer of nerve fibres is innermost and, thus, furthest removed from its oxygen source in the choroidal capillaries. This layer becomes increasingly hypoxic till, about four months in human gestation, mesenchymal cells invade the nerve fibre layer and differentiate to form the inner retinal vasculature which progresses peripherally towards the ora serrata $;{ }^{14}$ growth stops when pericyte-like mural cells become apposed to the capillaries. A circumferential avascular ring (the retinal vanguard) may persist in premature infants and this ring is a potential source of neovascularisation. Conversely, the peripheral capillaries are still immature and may be sensitive to hyperoxia which could cause their degeneration. ${ }^{12}$

Key words: angioblasts, angiogenesis, basement membrane, blood vessels, development, embryonic, endothelium, fibronectin, laminin

\section{References}

${ }^{1}$ Reagan FR: Vascularisation phenomena on fragments of embryonic bodies completely isolated from yolk sac entoderm. Am J Anat 1915, 9: 329-41.

${ }^{2}$ Noden DM: Origins and assembly of avian embryonic blood vessels. Ann NY Acad Sci 1990, 588: 236-49.

${ }^{3}$ Knower HME: Effects of early removal of the heart and arrest of the circulation on the development of frog embryos. Anat Rec 1907, 7: 161-5.

${ }^{4}$ Chapman WB: The effect of the heartbeat upon the development of the vascular system in the chick. Am J Anat 1918, 23: 175-203.

${ }^{5}$ Loeb J: Ueber die Entwicklung von Fischembryonen ohne kreislauf. Pflüg Arch 1893, 54: 525-31.

${ }^{6}$ Stockland CR: The origin of blood and vascular endothelium in embryos without a circulation of 
the blood and in the normal embryo. Am J Anat 1915, 18: 227-37.

${ }^{7}$ Sabin FR: On the origin of the cells of the blood. Physiol Rev 1922, 2: 38-69.

${ }^{8}$ Serpell G: Polysaccharide granules in association with developing retinal vessels and with retrolental fibroplasia. Br J Ophthal 1954, 38: 460-71.

${ }^{9}$ Wagner RC: Endothelial cell embryology and growth. Adv Microcirc 1980, 9: 45-75.

${ }^{10}$ Folkman $\mathrm{J}$ and Haudenschild $\mathrm{C}$ : Angiogenesis in vitro. Nature 1980, 228: 551-6.

${ }^{11}$ Hudlická O: Development of microcirculation: capillary growth and adaptation. In Renkin EM, Michel CC eds. Handbook of Physiology. The Cardiovascular System Volume IV. Bethesda: American Physiological Society 1984: 165-216.

${ }^{12}$ Hudlická O and Tyler KR: Angiogenesis: the growth of the vascular system. London: Academic Press. 1986.

${ }^{13}$ Grant DS, Kleinman HK, Martin GR: The role of basement membranes in vascular development. Ann NY Acad Sci 1990, 588: 61-72.

${ }^{14}$ Cogan DG, Kuwabara T, Friedman E: Retinal vasculature. Microvas Res 1968, 1: 115-32. 